\section{Perceived social dominance}

WAYNE WILSON and ROBERT LEHR, Stephen F. Austin State University, Nacogdoches, Tex. 75961

Seventy-four freshmen were asked to judge the concept of social dominance (or social submissiveness) by ranking a set of seven portraits of social scientists and by giving a definition of the concept. Results indicated significantly greater agreement among $S$ s on ranking portraits under the dominance criterion; also, submissiveness was defined less accurately, suggesting that this concept is not merely a reciprocal version of dominance.

Concepts are often defined by the $E$ on the presumption that his intended meaning will be embraced flawlessly by the $\mathrm{S}$. This practice may by symptomatic of the experimentalist's reputation for using the $S$ as a shadowy figure in research endeavors or, perhaps, the E simply feels secure in his ability to communicate clearly with almost any freshman or sophomore. Whatever the cause, there is a need to evaluate the degree of harmony one can expect between $E$ and $S$ concerning their mutual interpretation of concepts.

Perceived social behavior represents a ripe area for such research since the $S$ is usually called upon to use some criterion in judging a social setting devised by the $E$. For example, suppose Ss are instructed to rank a series of stylized portraits as being socially dominant or socially submissive. Supplying an explicit definition for these terms is no guarantee that a particular $S$ will adhere to the E's suggested connotation; in fact, the general familiarity of the concepts could influence him to alter the usage or to add to it. The present experiment is designed to emphasize the S's interpretation of social dominance or social submissiveness as contrasted with a "standard" (E-defined) definition of these terms. A secondary consideration involves an examination of the notion that dominance and submissiveness are bipolar concepts, with one being noted as the reciprocal of the other.

\section{METHOD}

The Ss were 74 students enrolled in freshman psychology courses during the summer and were required to participate in the experiment. Assignment of each student to one of four treatments involved a semirandom procedure with two constraints: First, each treatment reflected a like proportion of males to females; second, chance assignment was qualified by the stipulation that a comparable number of Ss be maintained in each treatment during testing.

The $E$ and $S$ sat opposite one another at a table in a quiet room. A 1-ft-high partition allowed the $E$ to hide his materials before presentation to the $S$. The materials were seven $8 \frac{1}{2} \times 11 \mathrm{in}$. stylistically designed portraits framed in a clear plastic cover, with seven portraits in color and an identical set of seven in black and white. The portraits were taken from the issues of Psychology Today, and more than one artist was involved in creating them, but all were full-page reproductions of social scientists against varying backgrounds. The men portrayed included Carl Rogers, Viktor Frankl, Peter Drucker, Michael Polanyi, Robert Wood, Abraham Maslow, and Henry Murray. With no disrespect intended, it should be noted that no $S$ recognized these individuals.

A 2 by 2 by 2 design was used with half the $S$ s instructed to rank the portraits in terms of social dominance and the remaining $S s$ instructed on the basis of social submissiveness. A second independent variable pertained to the presence or absence of color in the portraits. so included because it was one of the easier characteristics to control in assessing the impact of portrait features on the ranking judgments of Ss. A third variable under manipulation involved two forms of presentation: One approach entailed the method of paired comparisons, wherein each portrait was paired with every other portrait to produce a total of 21 combinations; a second manner of presentation was represented by a simple ranking procedure, with all portraits exposed to the $S$. The two methods were counterbalanced for order of presentation among all Ss and given separately in two sessions conducted 5 to 7 days apart.

The initial test session began for each $S$ with these instructions: "This is a task for which there are no right or wrong answers. I am going to show you some portraits of men who are completely unknown to you. I am interested in finding out how these men appear to you, based on the psychological trait of social dominance (social submissiveness)." For the method of paired comparisons, this information was given: "I will now place on the desk two portraits. From the time I lay them down, you will have 10 seconds to decide which of the two men is highest in social dominance (social submissiveness)." For the ranking method, the $E$ placed the portraits in a row on the desk, based on one of four random orders: "When you have decided which one appears to be highest and lowest in social dominance (social submissiveness), place the highest here (to the right of the $S$ ) and the lowest here (left of S). Now, of the ones you have left, which seems highest and lowest in social dominance (social submissiveness)? Place them here and here. Now, of these last three, which is highest and which is lowest? They also go here and here. This last one goes in the middle."

It should be pointed out that Ss were not given explicit definitions for dominance or submissiveness, nor were these concepts mentioned together to any $\mathrm{S}$. This practice was necessary, since, at the close of the second session, each $S$ was asked to respond to the following question: "Would you tell me what is the meaning of social dominance (social submissiveness) to you?" The E recorded each S's answer verbatim with the notion that a verbal reply might be less awkward than a written one.

In Appendix III of their paper, Dollard \& Mowrer (1947) reported a technique for analyzing written information into independent clauses for easier evaluation. This procedure was adopted by the present Es and applied to S-definitions of dominance and submissiveness. The rules of analysis are too lengthy for description here but details can be obtained on request; suffice to say that clauses were distributed among three categories bearing the labels of highly accurate definitions, relevant but approximate interpretations, and wholly inaccurate answers. These classifications were not weighted but simply used to organize frequency counts as to the number of clauses in each category. The S-definitions were judged against a commonly stated characteristic of the dominance concept, namely, the condition whereby one individual exercises priority or control over another individual in pursuit of a goal. In contrast, submissiveness has been defined typically as characterizing a person who yields to another and thereby relinquishes some desired objective.

\section{RESULTS AND DISCUSSION}

Kendall's coefficient of concordance (Siegel, 1956) was applied to data obtained from the two methods of ranking in order to determine the degree of agreement among $S s$ as to their dominant and submissive judgments of the seven portraits. Table 1 indicates clearly that all four coefficients under the dominance cirterion are substantially higher $(p<.01)$ than the nonsignificant values in the submissiveness category. Also, both ranking methods under the dominance label show greater $\mathrm{S}$ agreement with color than without it. 
Table 1

Concordance Coefficients of Ranking Judgments

\begin{tabular}{lccccc} 
& \multicolumn{2}{c}{ Dominance } & & \multicolumn{2}{c}{ Submissiveness } \\
\cline { 2 - 3 } \cline { 5 - 6 } Portrait & PC & RO & & PC & RO \\
\hline Color & $* .45$ & $* .40$ & .04 & .08 \\
No Color & $* .24$ & $* .32$ & .05 & .07 \\
\hline
\end{tabular}

$P C$ refers to paired comparisons method: $R O$ refers to rank order method.

* $p<.01$

Spearman's rank correlation coefficient (Siegel, 1956) was used to ascertain the reliability of the two ranking methods for the four treatments based on each of the seven portraits. A total of 28 coefficients were involved, with 19 occurring at the .01 level of significance, 7 at the .05 level, and 2 classified as not significant. The color-dominance group was most stable, with coefficients ranging from +.68 to +.82 ; least stable in agreement was the color-submissiveness group, which revealed a deviant value of -.10 for one portrait. Because the differing characteristics among portraits were not controlled (except for color), no effort was made to decipher the extreme disagreement between the ranking methods on this particular reproduction. On the whole, the range of correlational values would indicate moderate to good

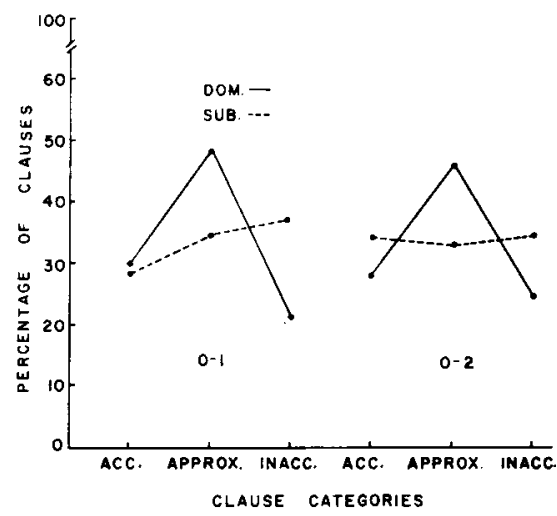

Fig. 1. 01 (junior author) and 02 show agreement that $\mathrm{Ss}$ defining submissiveness gave lower percentage of approximate definitions and $a$ higher percentage of wholly inaccurate answers. reliability for the ranking method with the method of paired comparisons.

The junior author and a naive $O$ were trained to apply Dollard and Mowrer's clause analysis to each S's definition, as classified under either a dominant or submissive rubric. Figure 1 illustrates the comparable results of the two judges, showing that Ss describing submissiveness produced fewer approximate definitions and more inaccurate interpretations than did Ss defining the dominance concept The two Os yielded a small disparity in the number of clauses claimed, with the junior author tabulating 235 and the naive $\mathrm{O}$ a total of 224

Actually, the degree of agreement shown in dominance rankings does not imply that portrait judgments were valid, only that they were reliable. The data in Table 1 suggest that the addition of color provided a $S$ with more information from which to form a judgment, but it is not known how other portrait features may have contributed to a S's decision. It is possible that the portraits may have been more expressive of dominance than of submissiveness, or that the diversity of figure-to-ground relationships among the portraits still did not permit an adequate range of dominant-submissive characteristics from which genuine rankings could be obtained.

Another indeterminant factor concems the number of stimuli that a $S$ can reasonably be expected to recognize and rank; linear infrahuman dominance orders lose their definition and are rearranged in various patterns when group size exceeds a certain number. Presumably a failure to recognize all the members facilitates a different hierarchy, indicating that a comparable change should occur under human social perception when Ss are confronted with a greater number of variables (portraits, people, ideas) to evaluate and rank than they can assimilate. Some kind of order could be generated, to be sure, but it would be necessary to ascertain, for example, if the intermediate ranks were artifactual or if the $S$ had truly been able to discriminate among them.
Accounting for these parameters will not reduce the number of probable alternative hypotheses to one or two; there remains another source of variance in terms of the dominance or submissiveness criterion applied by each $\mathrm{S}$. Although no clear estimate of validity was available for portrait judgments, a standard definition of dominance (exercising priority) and submissiveness (yielding) was established for comparison with S-definitions. Inspection of Fig. 1 prompts the conclusion that interpretations of submissiveness were less accurate, possibly because this concept simply is less structured or that it encompasses fewer associations than does dominance, possibly because any term that carries a negative connotation may be less prominent in one's vocabulary.

Even so, it is difficult to refrain from questioning the status of submissiveness as a reciprocal version of dominance. Assuming that "dominant" is the more familiar (positive) adjective in a bipolar context, one could argue that "submissive" would be defined as "that which dominance is not." However, unipolar definitions of submissiveness in the present experiment do not suggest that this interpretation was readily apparent to Ss. If a particular connotation of submissiveness is sharpened in one format but vague in another, there is the likelihood that a comprehensive understanding of the term cannot be realized in a single context.

Granting the premise that covert and overt expressions were isomorphic for each concept, it is evident that judgments were less valid for submissiveness than for dominance. No matter how clearly submissive features may have been differentiated by the seven portraits, the $S$ 's interpretation still would have suffered by virtue of an ambiguous criterion.

$$
\text { REFERENCES }
$$

DOLLARD, J., \& MOWRER, O. H. A me thod of measuring tension in written documents. Journal of Abnormal \& Social Psychology, $1947,42,3-32$

SIEGEL, S. Nonparametric statistics. New York: McGraw-Hill, 1956. 